\title{
L'Association Italienne de Myologie (AIM) : Avanti Miologia
}

\section{Gabriele Siciliano}

\begin{abstract}
L'Association Italienne de Myologie (AIM) est une société savante fondée en 2000 dans le but de mettre en place un réseau national destiné à améliorer la connaissance et les efforts cliniques et scientifiques dans le domaine des maladies musculaires. Elle encourage les collaborations entre groupes, particulièrement dans le cadre d'études multicentriques et pluridisciplinaires. De plus, elle soutient des échanges culturels, scientifiques et des relations avec des Associations Européennes et Internationales similaires. À ce jour, 150 membres et quatre membres honoraires constituent l'AIM, la plupart d'entre eux travaillant dans 30 centres cliniques et pluridisciplinaires ouverts à tout professionnel du domaine des maladies neuromusculaires et des systèmes de santé.
\end{abstract}

L'association italienne de myologie (AIM ; www.miologia.org) fut fondée en 2000 à l'initiative de 64 membres fondateurs partageant un intérêt commun pour les maladies du muscle.

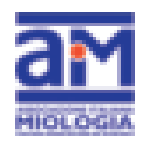

Ces pionniers décidèrent de construire un réseau national en vue d'améliorer les connaissances et promouvoir les efforts cliniques et scientifiques dans ce domaine. Dès le début, l'association a vu le jour sous l'égide de la Société Italienne de Neurologie, à laquelle elle est toujours connectée. Au fil du temps elle a souhaité inclure des personnalités variées telles que des neuropédiatres, des scientifiques, des cardiologues, pneumologues, psychologues et des spécialistes de neuro-rééducation. La myologie concentrait en effet un intérêt croissant vers des maladies ayant bénéficié de la révolution générée par les découvertes spectaculaires moléculaires et génétiques des années 1980, grâce notamment au développement des technologies d'ADN recombinant. Ceci a provoqué une nouvelle vague d'enthousiasme chez ceux qui, suite aux travaux des groupes pionniers de J.Walton, V. Dubowitz, A. Engel, M. Brooke, V. Askanas, et plus récemment M. Fardeau, S. Di Mauro, C. Angelini, et G. Nigro, pour ne citer qu'eux, ont entrepris de travailler dans ce domaine.

De nos jours, la myologie a permis d'avancer dans tous les domaines allant du diagnostic, tant moléculaire que clinique, à la neurophysiologie, l'anatomo-pathologie et l'imagerie musculaires, jusqu'aux approches thérapeutiques grâce à une activité de recherche très soutenue. Cette émulation a donné lieu, en Italie, à un effort collaboratif entre myologues afin d'améliorer leurs activités respectives, tout en contribuant à mettre en contact les scientifiques et plusieurs associations de patients impliquées dans ce domaine. Cela a permis, l'an passé, de mettre en place une instance de coordination, la CAMN (coordination de l'association des maladies neuromusculaires), qui est devenu une interface privilégiée pour des initiatives dédiées à la rédaction de recommandations et directives, de protocoles de prise en charge des patients, l'organisation de registres de maladies et, à terme, la mise en place d'essais thérapeutiques.

Globalement, les objectifs principaux de l'AIM sont : - d'établir, au niveau national, une référence scientifique pluridisciplinaire pour la promotion et la dissémination de la connaissance dans les domaines neuromusculaires, au bénéfice des patients ;

- de stimuler et encourager le développement de protocoles cliniques et la rédaction de recommandations pour le diagnostic et la thérapeutique (NDLR ; l'équivalent de nos PNDS);

- d'apporter un soutien et des opportunités pour les jeunes travaillant dans le domaine neuromusculaire ; - d'organiser des conférences nationales et internationales ;

- d'encourager les collaborations entre les différentes équipes, particulièrement dans le cadre d'études multicentriques et pluridisciplinaires, et de soutenir les avancées de la recherche clinique et "fondamentale ", en complément des associations scientifiques tournées vers les maladies génétiques au sens large, comme le Telethon-I, et dans d'autres disciplines ;

- de présenter les personnes intéressées aux instances des institutions académiques, ministérielles et des services de santé afin de définir des stratégies éducatives et économiques, surtout du fait que les maladies neuromusculaires sont reconnues comme 
maladies rares. 15 établissements de soins italiens appartiennent désormais au Réseau Européen de Référence Euro-NMD récemment labellisé ;

- d'établir des échanges culturels, scientifiques et des modes de communications pratiques avec des associations européennes et internationales de même type.

Ces objectifs sont atteints par l'organisation de rencontres régulières générales au niveau national, dont une au printemps, et de plusieurs autres sur des thèmes précis au niveau régional, afin d'échanger des informations scientifiques et de promouvoir la formation et l'éducation des jeunes médecins et/ou chercheurs. Le journal scientifique officiel de l'association est Acta Myologica (Éditeur Conte Academy, ISSN 1128-2460).

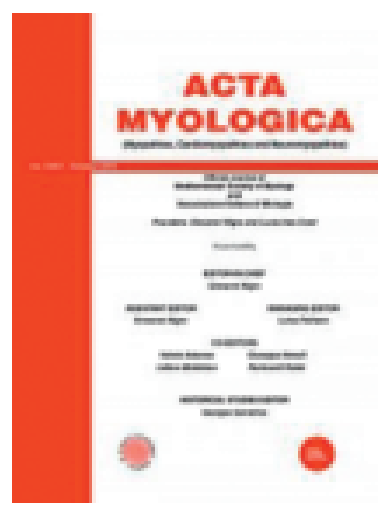

L'AIM est organisée autour d'un comité exécutif de direction composé du président, de l'ex-président et de huit membres du conseil élus pour trois ans lors d'une assemblée générale. Leur mandat ne peut être renouvelé qu'une seule fois. À ce jour, $150 \mathrm{mem}$ bres et 4 membres honoraires composent l'AIM. Ils sont répartis dans 30 centres cliniques pluridisciplinaires, avec des outils de laboratoire, principalement localisés dans des universités et deux centres plus spécialisés qui font office de centres de références pour les études génétiques, ouverts à tous les professionnels du domaine des maladies neuromusculaires et des soins. Les centres cliniques et les laboratoires interagissant en réseau avec l'AIM sont répartis sur tout le territoire italien, du nord au sud.

The Italian Association of Myology (AIM): Avanti Miologia

\section{LIENS D'INTÉRÊT}

L'auteur déclare n'avoir aucun lien d'intérêt concernant les données publiées dans cet article.

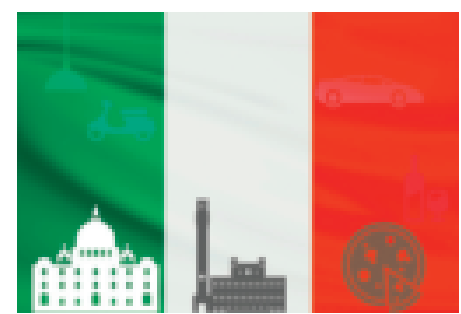

Des myologues italiens de renom

- Les Anglais ne sont pas les seuls à disputer aux Français, et en particulier à Duchenne de Boulogne, la paternité des premières descriptions de la dystrophie musculaire. Gaetano Conte semble aussi, d'après nos amis italiens, l'avoir précédé de plusieurs années (en 1836 pour être précis).

- D'autres noms de chercheurs italiens sont également passés à la postérité de la myologie : Vialetto, Fazio et Londe ont particulièrement œuvré, par exemple, dans le domaine des amyotrophies bulbo-spinales, etc.

- Quant à Salvatore di Mauro, et malgré son expatriation aux Etats-Unis, il reste le champion incontesté des myopathies métaboliques et mitochondriales.

- Depuis janvier 2013, le Docteur Zeviani est professeur-chercheur à l'Université de Cambridge au Royaume-Uni et a pris la suite de Sir John Walker, Prix Nobel de Chimie en 1997, à la tête de l'unité de biologie mitochondriale au sein du MRC (Medical Research Council).

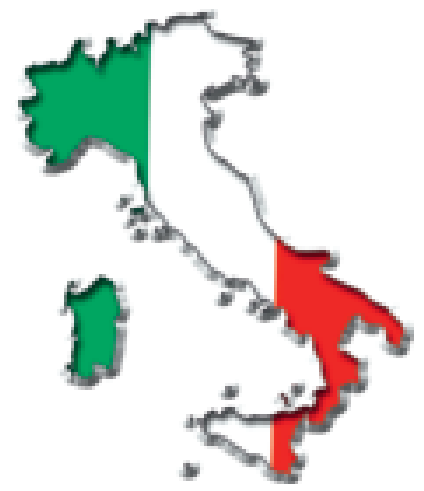

